\title{
Performance indicators for monitoring the integrated care pathway of patients with colorectal cancer: the E.Pic.A. project
}

\author{
Andrea Ricotti ${ }^{1}$, Giovanni Messori Ioli ${ }^{1}$, Mattia Altini ${ }^{2}$, Letizia Bachini ${ }^{3}$, William Balzi ${ }^{2}$, Giovanni L. Frassineti ${ }^{2}$, \\ Fabrizio Gemmi ${ }^{3}$, Ilaria Massa ${ }^{2}$, Andrea Pierini ${ }^{4}$, Oscar Bertetto ${ }^{5}$, Patrizia Racca ${ }^{6}$ \\ ${ }^{1}$ Azienda Sanitaria Locale CN2, Alba - Italy \\ ${ }^{2}$ Istituto Scientifico Romagnolo per lo Studio e la Cura dei Tumori (IRST) IRCCS, Meldola - Italy \\ ${ }^{3}$ Tuscan Regional Health Agency, Florence - Italy \\ ${ }^{4}$ Roche S.p.A., Monza - Italy \\ ${ }^{5}$ Dipartimento Interaziendale Interregionale Rete Oncologica Piemonte-Valle d'Aosta, Torino - Italy \\ ${ }^{6}$ A.O.U. Citta della Salute e della Scienza di Torino, Torino - Italy
}

\begin{abstract}
Background: Given limitations in economic resources, achieving sustainability in healthcare is an increasingly important issue driving policy decisions. Colorectal cancer (CRC) is a frequent oncological diagnosis with an incidence that is expected to increase, making the efficient utilisation of resources a major priority for its diagnosis and treatment. To assess potentially inappropriate utilisation of services in the integrated care pathway of patients with malignant CRC, a series of key performance indicators (KPIs) have been developed by a multidisciplinary panel of the E.Pic.A. project (Economic Appropriateness of an Integrated Care Pathway).

Methods: The KPIs identified had to comply with criteria of reliability, representativeness, accessibility and operativity. A definition is provided for each of the KPIs, along with the methodology used to calculate it and a reference target.

Results: Eight KPIs were identified that can measure inadequacies of services provided in the diagnostic and treatment pathways for CRC: four for instrumental diagnostics, three for surgery and one for oncologic therapy. Conclusions: Use of the methodology described can help payors to obtain detailed information on inappropriate and wasteful use of healthcare resources, which would then permit their reallocation for interventions with higher value for patients with CRC and other pathologies.
\end{abstract}

Keywords: Appropriateness, Colorectal cancer, Italian National Health System, Key performance indicators, Sustainability

\section{Introduction}

The sustainability of the Italian National Health System (INHS) is one of the most important issues driving policy decisions in healthcare. At least in the short term, resources for healthcare are not expected to increase and remain limited compared to those of other countries of similar economiccultural standing. Indeed, "times of continuous economic growth and the broadening of social protection of the public seem, if not lessened, at least temporarily set aside and the INHS cannot ignore this" (1). It is thus necessary to guarantee

Submitted: May 17, 2019

Accepted: May 23, 2019

Published online: June 15, 2019

\author{
Corresponding author \\ Andrea Ricotti \\ Azienda Sanitaria Locale CN2, Alba \\ Via Vida 10, 12051 Alba, CN, Italy \\ 189290@edu.unito.it
}

the sustainability of the INHS until action in prevention and innovation can compensate for the increased burden related to progressive ageing of the general population (2). In this regard, a recent report has highlighted potential areas (overand under-treatment, purchases at excessive costs, administrative efficiency, administrative complexities, frauds and abuses, etc.) that can be drawn upon to free up resources that can be reinvested or reallocated (3). Considering this scenario, evaluation of healthcare performance has gradually become an increasingly important activity for governance of the INHS (4). The analysis of integrated care pathways by defining key performance indicators (KPIs) is an effective tool that the INHS can utilise for this purpose (5).

Colorectal cancer (CRC) is the most frequent oncological diagnosis in Italy with a frequency of $14 \%$ (16\% in males and $13 \%$ in females) and is the second cause of oncologic death (11\% in males, $12 \%$ in females) (6). There is a slight tendency towards decreased mortality and increasing incidence; the latter is mainly linked to earlier diagnosis of CRC (in 2017, approximately 53,000 new diagnoses of CRC were estimated; $+1.1 \%$ compared to 2016) (6). Considering the positive trend in survival observed in recent years, in the near future it can be expected 
that there will be constant growth in the need for healthcare resources, making the efficiency of healthcare investments required for the integrated care pathway of CRC a top priority.

With a view towards rendering healthcare resources more efficient, it is essential to measure the performance of various activities used for the integrated care pathway of CRC. Herein, the methodology developed by a multidisciplinary panel of the E.Pic.A. project (Economic Appropriateness of an Integrated Care Pathway) is described with the aim of assessing potentially inappropriate behaviour, from clinical and economic standpoints, of various processes in the integrated care pathway of patients undergoing intervention for carcinoma of the colon or rectum.

\section{Methods}

Following the recent Associazione Italiana di Oncologia Medica guidelines for the treatment of CRC (7), the multidisciplinary panel of the E.Pic.A. project (composed of clinicians, healthcare directors, data managers, methodologists and experts) identified and defined a series of KPIs that are suitable for assessing potentially inappropriate healthcare services provided during diagnosis and treatment of patients undergoing surgical intervention for malignant CRC. In order for the evaluation process to be consistently replicated by each healthcare facility, the CRC KPIs must be easily detectable and measurable through the most common information flows currently available (e.g. administrative databases, hospital discharge records [HDRs], etc.).

In order to maximise the efficiency of healthcare services offered to patients, each of the KPIs identified must comply with the criteria of "reliability" (fewest possible systematic errors), "representativeness" (high degree of relationship with the pathway in question), "accessibility" (directly measurable by the available information sources) and "operativity" (must be easily applicable in the context under assessment) (8). Lastly, for each of the KPIs identified, the multidisciplinary panel: (i) provided a definition; (ii) explained the methodology for its calculation; and (iii) indicated a reference target for evaluation.

\section{Results}

Altogether, eight KPIs were identified to measure the inappropriateness of healthcare services provided in the integrated care pathway for CRC: four for instrumental diagnostics, three for surgery and one for oncologic therapy. The four KPIs for the diagnostic phase aimed to estimate the inappropriateness of diagnostic procedures (under-treatment and over-treatment) before and after the index surgical intervention. The three KPIs for the surgical phase measured the timeliness of the index surgery, the hospitalisation associated with it and any re-interventions carried out during the same hospitalisation. For oncological therapy, the last KPI measured the inappropriateness referred to the time of administration of adjuvant therapy (chemotherapy and/or radiotherapy) vs. the time elapsed following the index surgery.

Unless otherwise specified, each of the eight KPIs must be calculated by distinguishing between patients undergoing intervention for carcinomas of the colon and those of the rectum. Herein, for simplification, whenever the KPI is calculated for a patient with colon cancer or rectal cancer, the term "surgical intervention for CRC" will be used; otherwise, if the KPI is calculated with reference to only the patient undergoing intervention for colon cancer or for rectal cancer, the relative diagnosis will be specified.

Since in both cases (colon and rectum), the date of the index surgical procedure (and the relative hospitalisation index) represents the reference time for calculation of each of the eight KPIs, it is fundamental to provide a definition that also includes the identification criterion. Firstly, in order to identify new patients undergoing surgery each year for malignant CRC, the multidisciplinary panel established that the reference time frame for surgical interventions should be the calendar year (January 1 to December 31 ). In the case of colon cancer, the index surgery was defined as the first admission for surgery in the year of observation characterised by the simultaneous presence of one of the diagnostic codes and one of the procedural codes listed in Table I

Table I - Colorectal ICD-9-CM procedural codes for diagnosis and various interventions

\begin{tabular}{|c|c|}
\hline \multicolumn{2}{|l|}{ Colon } \\
\hline ICD-9-CM code & Diagnosis \\
\hline $153^{*}$ & Malignant tumour of the colon \\
\hline ICD-9-CM code & Procedure \\
\hline 45.71 & $\begin{array}{l}\text { Multiple segmental resection of the large inte- } \\
\text { stine }\end{array}$ \\
\hline 45.72 & Resection of the caecum \\
\hline 45.73 & Right hemicolectomy \\
\hline 45.74 & Resection of the transverse colon \\
\hline 45.75 & Left hemicolectomy \\
\hline 45.76 & Sigmoidectomy \\
\hline 45.79 & Other partial excision of the large intestine \\
\hline 45.8 & Total colectomy \\
\hline
\end{tabular}

\section{Rectum}

ICD-9-CM code

Diagnosis

154.0

Malignant tumour of the rectosigmoid junction

$154.1 \quad$ Malignant tumour of the rectum

$154.8 \quad$ Other malignant tumours of the rectum, rectosigmoid junction and anus

\section{ICD-9-CM code Procedure}

48.3* Local excision of lesion or rectal tissue

48.4* Rectal pull-through resection

$48.5 \quad$ Abdominoperineal resection of the rectum

48.6* Other resection of the rectum

$48.74 \quad$ Proctectomy

$68.8 \quad$ Pelvic exenteration

48.99

Other intervention on the rectum and peri-rectal tissues 
(information flow produced by the HDRs) for colon cancer. With the index surgical procedure thus identified, the following must be excluded:

- patients under the age of 18 at the time of index admission;

- patients present in the registry for less than 3 years from the date of the index admission;

- cases with at least one hospitalisation for colon cancer (according to the International Classification of Diseases 9th revision Clinical Modification ICD-9-CM 153*) in the main or secondary diagnosis for a period between 3 years and 6 months prior to the date of surgery performed during the index hospitalisation;

- cases with other neoplasia and at least one hospitalisation for a malignancy other than the colon (ICD-9-CM $140 *-152 *$ or $154 *-172 *$ or $174 *-208 *$ or V.10* excluding V10.05) in the main or secondary diagnosis for 3 years prior to the date of surgery performed during the index hospitalisation;

- patients with ab initio metastatic disease (ICD-9-CM $197 *, 198 *$ and 199*) during the index admission.

In the case of rectal carcinoma, the index surgical procedure was defined as the first admission for surgery in the year of observation characterised by the simultaneous presence of one of the diagnostic codes and one of the procedural codes listed in Table I (information flow produced from HDRs) for rectal cancer. From the index surgical procedures thus identified, the following should be excluded:

- patients under the age of 18 at the time of index admission;

- $\quad$ patients present in the registry for less than 3 years from the date of the index admission;

- cases with at least one hospitalisation for rectal cancer (ICD-9-CM V.10.06 or 154*) in the main or secondary diagnosis for a period between 3 years and 6 months prior to the date of surgery performed during the index hospitalisation;

- cases with other neoplasia and at least one hospitalisation for a malignancy other than rectal (ICD-9-CM 140*_ $153^{*}$ or $155^{*}-172 *$ or $174 *-208^{*}$ or V.10* excluding V10.06) in the main or secondary diagnosis for 3 years prior to the date of surgery performed during the index hospitalisation;

- patients with ab initio metastatic disease (ICD-9-CM $197 *, 198 *$ and 199*) during the index admission.

\section{KPIs for instrumental diagnosis}

\section{KPI-1 pre-surgery 1}

Definition. The first KPI evaluates the percentage of patients undergoing surgical intervention for CRC who, in the 60 days prior to the index surgery, underwent at least one of the following instrumental diagnostic procedures: positron emission tomography (PET) or bone scintigraphy. Patients who underwent computerised axial tomography (CAT) within 90 days (indication of the multidisciplinary panel) prior to the execution of one of the two diagnostic procedures considered are excluded from evaluation, since the use of PET or bone scintigraphy is to be attributed to diagnostic uncertainty highlighted by a previous CAT scan. Table II shows the codes to be used to identify instrumental diagnostic examinations, as defined by the E.Pic.A. multidisciplinary panel.

Target. The percentage for $\mathrm{KPI}-1$ should tend to be $0 \%$.

Calculation. The percentage should be calculated by indicating in the numerator the number of patients undergoing surgical intervention for CRC who underwent at least one PET and/or bone scintigraphy in the 60 days prior to the index surgery (codes shown in Tab. II). Patients who underwent a CAT scan (Tab. II) in the 90 days prior to the date of at least one of the two diagnostic procedures considered should be excluded. The denominator is the number of patients undergoing intervention (index surgery) for CRC during the reference period.

\section{$\mathrm{KPI}-2$ pre-surgery 2}

Definition. The second KPI assesses the percentage of patients undergoing intervention for rectal carcinoma who, in the 112 days prior to the index surgery, underwent at least one of the following instrumental diagnostic examinations: pelvic nuclear magnetic resonance imaging (MRI) or transrectal ultrasound. The identification codes associated with these examinations are shown in Table II, as defined by the E.Pic.A. multidisciplinary panel.

Target. The percentage of KPI-2 should tend to be $100 \%$.

Calculation. The percentage must be calculated by indicating in the numerator the number of patients undergoing intervention for rectal cancer who underwent at least one pelvic MRI and/or transrectal ultrasound (codes in Tab. II) in the 112 days prior to the index surgery. The denominator is the number of patients undergoing intervention (index surgery) for carcinoma of the rectum during the reference period.

The third KPI was sub-divided into KPI-3a and KPI-3b.

\section{KPI-3a follow-up colonoscopy}

Definition. KPI-3a evaluates the percentage of patients undergoing intervention for colon cancer who, during the 365 days following the index surgery, underwent colonoscopy. Table II shows the codes to be used to identify colonoscopy examinations, as defined by the multidisciplinary panel.

Target. The percentage of KPI-3a should tend to be $100 \%$.

Calculation. The percentage must be calculated by indicating in the numerator the number of patients undergoing intervention for colon cancer who underwent colonoscopy (Tab. II) during the 365 days following surgery. The denominator refers to the number of patients (index surgery) undergoing surgery for colon cancer in the reference period.

\section{KPI-3b follow-up colonoscopy}

Definition. KPI-3b evaluates the percentage of patients undergoing intervention for colon cancer in whom, during the 365 days following the index surgery, more than one colonoscopy was performed more than 30 days from the first colonoscopy. This time span was considered as appropriate to indicate that the second colonoscopy, taking place within 30 days of the prior one, is to be attributed to the "tattooing" of a possible recurrence or to confirmation of a previously 
Table II - Codes for instrumental diagnoses and tumour markers PET

92.11.6 - PET (qualitative)

92.11.7 - PET (quantitative)

92.18.6 - Total body PET

\section{Bone scintigraphy}

92.05.6 - Total body bone marrow scintigraphy

92.14.1 - Bone scintigraphy articular segment

92.14.2 - Bone scintigraphy polyphasic articular segment

92.14.3 - Segmental skeletal tomoscintigraphy (SPET)

92.18.2 - Bone or articular scintigraphy

CT

88.01.3 - Lower abdomen CT

88.01.4 - Lower abdomen CT with and without contrast

88.01.5 - Complete abdominal CT

88.01.6 - Complete abdominal CT with and without contrast

\begin{tabular}{|c|c|}
\hline & NMR \\
\hline & 88.95.4 - Lower abdomen and pelvic MRI \\
\hline & $\begin{array}{l}\text { 88.95.5 - Lower abdomen and pelvic MRI with and } \\
\text { without contrast }\end{array}$ \\
\hline & Transrectal ultrasound \\
\hline 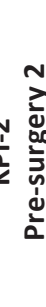 & 88.79.8 - Transrectal ecography \\
\hline
\end{tabular}

\begin{tabular}{|c|c|}
\hline \multirow{4}{*}{ 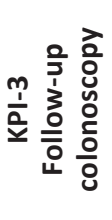 } & Colonoscopy \\
\hline & 45.23 - Colonoscopy with flexible endoscope \\
\hline & 45.23.1 - Colonoscopy - retrograde ileoscopy \\
\hline & 45.24 - Sigmoidoscopy with flexible endoscope \\
\hline
\end{tabular}

\begin{tabular}{|c|c|}
\hline \multirow{3}{*}{ 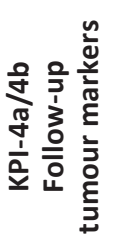 } & Tumour markers \\
\hline & 90.55.3-CA 19.9 \\
\hline & $90.56 .3-C E A$ \\
\hline
\end{tabular}

$\mathrm{CA}=$ carbohydrate antigen; $\mathrm{CEA}=$ carcinoembryonic antigen $; \mathrm{CT}=$ computed tomography; $\mathrm{KPI}=$ key performance indicator; $\mathrm{MRI}=$ magnetic resonance imaging; NMR = nuclear magnetic resonance; $\mathrm{PET}=$ positron emission tomography; SPET = Single Photon Emission Computed Tomography.

observed lesion. Table II shows the codes to be used to identify colonoscopy examinations, as defined by the multidisciplinary panel.

Target. The percentage of KPI-3b should tend to be $0 \%$.

Calculation. The percentage should be calculated by indicating in the numerator the number of patients undergoing intervention for colon cancer who underwent more than one colonoscopy (Tab. II) during the 365 days following surgery within a time frame of more than 30 days between the two diagnostic procedures. The denominator refers to the number of patients (index surgery) undergoing intervention for colon cancer during the reference period.

The fourth KPI was sub-divided into KPI-4a and KPI-4b.

\section{KPI-4a follow-up tumour marker 1}

Definition. In reference to the year following the index surgery, KPI-4a evaluates the annual percentage of patients undergoing intervention for CRC who had the same tumour marker evaluated more than three times (7), considering the markers indicated by the multidisciplinary panel (Tab. II).

Target. The percentage of $\mathrm{KPI}-4 \mathrm{a}$ should tend towards $10 \%$.

Calculation. The annual percentage should be calculated by indicating in the numerator the number of patients who had the same tumour marker evaluated more than three times in the first year after CRC surgery (7) among those indicated in Table II. The denominator is the number of patients (index surgery) undergoing intervention for CRC during the reference period.

\section{KPI-4b follow-up tumour marker 2}

Definition. With reference to the two years following the index surgery, $\mathrm{KPI}-4 \mathrm{~b}$ evaluates the percentage of patients undergoing intervention for $\mathrm{CRC}$ who had both markers listed in Table II simultaneously evaluated at least once.

Target. $\mathrm{KPI}-4 \mathrm{~b}$ should tend to be $0 \%$ since evaluation of both markers is not supported by clinical evidence, and use of both markers does not represent an improvement for diagnostic purposes.

Calculation. The percentage should be calculated by indicating in the numerator the number of patients who, in the two years after CRC surgery, had both markers indicated by the multidisciplinary panel simultaneously evaluated at least once (Tab. II). The denominator is the number of patients undergoing intervention (index surgery) for CRC during the reference period.

\section{Surgical KPIs}

\section{KPI-5 time elapsed between biopsy and surgery}

Definition. The fifth KPI assesses the percentage of patients undergoing intervention for CRC who underwent surgery within 60 days of biopsy if not a candidate for neoadjuvant therapy (chemotherapy or radiotherapy), or within 90 days after biopsy if candidate for neoadjuvant therapy. Table III shows the codes to be used to identify biopsy and neoadjuvant therapy (chemotherapy or radiotherapy), as defined by the multidisciplinary panel.

Target. The percentage of KPI- 5 should tend towards $100 \%$.

Calculation. The percentage should be calculated by indicating in the numerator the number of patients with CRC who were subjected to biopsy and who underwent surgery within 60 days if not a candidate for neoadjuvant therapy, or within 90 days if candidate for neoadjuvant therapy, after the date of the biopsy. The denominator is the number of patients diagnosed with CRC who underwent biopsy and index surgery in the reference period. Patients with colon cancer who presented with a code for metastasis must be excluded: 196.* 
Table III - Biopsy and neoadjuvant therapy (chemotherapy or radiotherapy) codes identified by E.Pic.A

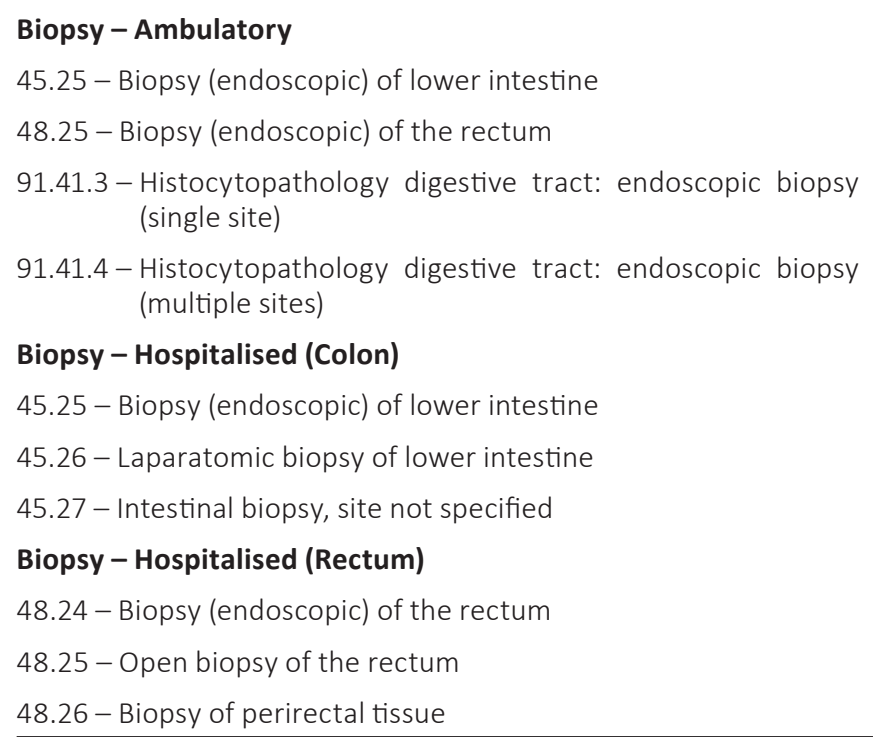

\section{Codes for Chemotherapy}

SPA database (Specialist Outpatient Assistance)†

99.25 - Injection or infusion of chemotherapy for tumour

99.25.4 - Antitumoural therapy with infusion of drug

99.25.5 - Antitumoural therapy with oral drugs or IM or SC injection

$8901 \mathrm{F0}$ - Antitumoural therapy with oral drugs or IM or SC injection excluding costs of drugs used for therapy per session (cycle with up to 30 sessions)

99.25.01 - Antitumoural therapy with infusion of drug - excluding costs of drugs used for therapy per session (cycle with up to 30 sessions)

From hospital discharge sheets

Admissions with diagnosis (all positions) V58.1*, codes (all positions) 99.25 and diagnosis-related group of 410

V58.11 - Antineoplastic chemotherapy

V58.12 - Antineoplastic immunotherapy

99.25 - Injection or infusion of chemotherapy for tumour

$410 \mathrm{M}$ - Chemotherapy not associated with secondary diagnosis of acute leukaemia

Hospitalisations with diagnosis (all positions) V580* and intervention codes (all positions)

Interventional radiotherapy codes

92.20 - Radioisotope infusion for brachytherapy

92.21 - Surface radiation

92.22 - Orthovoltage radiation

92.23 - Radioisotope teleradiotherapy

92.24 - Teleradiotherapy with photons

92.25 - Teleradiotherapy with electrons

92.26 - Teleradiotherapy with other particle radiations

92.27 - Implantation or insertion of radioactive elements
92.28 - Injection or instillation of radioisotopes

92.29 - Other radiotherapeutic procedures

92.30 - Stereotactic radiosurgery

92.31 - Single-source photonic radiosurgery

92.32 - Multi-source photonic radiosurgery

92.33 - Proton beam therapy

92.39 - Stereotactic radiosurgery

\section{ASA - SPA - Flow C: Radiotherapy codes}

92.23.2 - Telecobalt therapy multiple fields, moving

92.24.01 - Teletherapy with linear accelerator with multiple fields or movement for 3D technique

92.24.02 - Teletherapy with linear accelerator with multiple fields or movement with modulation of intensity

92.24.1 - Teletherapy with linear accelerator fixed field

92.24.2 - Teletherapy with linear accelerator with multiple fields, moving

92.24.3 - Teletherapy with linear accelerator flash technique

92.24.B - Radiotherapy with linear accelerator with MLC for IMRT static or dynamic multiple fields or moving

92.25.1 - Electron beam teletherapy with one or more fixed fields

92.25.2 - Total skin electron irradiation (TSEI/TSEBI)

ASA = Outpatient care; E.Pic.A. = Economic Appropriateness of an Integrated Care Pathway; IM = intramuscular; SC = subcutaneous; IMRT = Intensity Modulated Radiotherapy; MLC = Multileaf Collimator; SPA = Outpatient specialist care; TSEI = Total skin electron irradiation; TSEBI = Total Skin Electron Beam Irradiation.

†All codes referable to 99.25 , where present, are included. All identification codes for chemotherapy under a ADS (Ambulatory Day Service) regime, to be introduced starting from 2016, must be included (all regions that are not part of the pilot project must integrate with appropriate reference codes).

(exclude 196.2), 197.* and 198.*.

\section{KPI-6 duration of hospitalisation}

Definition. The sixth KPI assesses the percentage of patients undergoing intervention for CRC with duration of hospitalisation for surgery $>10$ days (7), if the diagnosis-related group (DRG) associated with the index admission is not complicated, or beyond 15 days (indication of the multidisciplinary panel) if the DRG is complicated, as defined in Table IV by the multidisciplinary panel.

Target. The percentage of KPI-6 should tend towards $10 \%$.

Calculation. The percentage should be calculated by indicating in the numerator the number of patients undergoing intervention for CRC whose hospitalisation for surgery extends beyond 10 days if the DRG is not complicated, or more than 15 days if the DRG is considered complicated. The denominator is the number of patients (index surgery) undergoing surgical intervention for CRC during the reference period.

\section{$\mathrm{KPI}$-7 subsequent re-intervention}

Definition. The seventh KPI evaluates the percentage of patients undergoing intervention for CRC with a second re-intervention following the index surgical procedure performed during the same index hospitalisation. The following 
Table IV - DRG codes identified by E.Pic.A

\section{Colon}

DRG149 - Major intervention on large and small intestines without complications

DRG569 - Major interventions on large and small intestines with complications with $\mathrm{Gl}$ diagnosis

DRG570 - Major interventions on large and small intestines with complications without $\mathrm{Gl}$ diagnosis

DRG541 - Extracorporeal membrane oxygenation or tracheostomy with mechanical ventilation $\geq 96$ hours or main diagnosis not related to face, mouth and neck with major surgical intervention

DRG542 - Tracheostomy with mechanical ventilation $\geq 96$ hours or main diagnosis not related to face, mouth and neck without major surgical intervention

Rectum

DRG146 - Rectal resection with complications

DRG147 - Rectal resection without complications

DRG149-Major interventions on large and small intestines without complications

DRG569 - Major interventions on large and small intestines with complications with $\mathrm{Gl}$ diagnosis

DRG570 - Major interventions on large and small intestines with complications without $\mathrm{Gl}$ diagnosis

DRG541 - Extracorporeal membrane oxygenation or tracheostomy with mechanical ventilation $\geq 96$ hours or main diagnosis not related to face, mouth and neck with major surgical intervention

DRG542 - Tracheostomy with mechanical ventilation $\geq 96$ hours or main diagnosis not related to face, mouth and neck without major surgical intervention

DRG = diagnosis-related group; E.Pic.A. = Economic Appropriateness of an Integrated Care Pathway; $\mathrm{GI}$ = gastrointestinal.

ICD-9-CM codes were used to identify re-intervention procedures:

- ICD-9-CM 45.* (incision, removal and anastomosis of the intestine),

- ICD-9-CM 46.* (other interventions on the intestine),

- ICD-9-CM 47.* (interventions on the appendix),

- ICD-9-CM 48.* (interventions on the rectum, rectosigmoid and peri-rectal tissues).

Target. The percentage KPI-7 should not exceed $5 \%$.

Calculation. The percentage should be calculated by indicating in the numerator the number of patients with reintervention, following the index intervention, and performed during the same index hospitalisation. The denominator is the number of patients (with index surgery) undergoing intervention for CRC during the reference period.

\section{KPI for oncological therapy}

\section{$\mathrm{KPI}-8$ therapy}

Definition. The last KPI assesses the percentage of patients undergoing intervention for $\mathrm{CRC}$ who, as candidates for adjuvant therapy (chemotherapy or radiotherapy), initiate adjuvant therapy within 56 days of the index surgery (7). The strategy defined to calculate $\mathrm{KPI}-8$ focuses on identification of all chemotherapy and/or radiotherapy administrations delivered after the date of the index surgical intervention during hospitalisation or in an outpatient regimen. Table III reports the sources and codes to identify the administration of chemotherapy and radiotherapy.

Target. The percentage of KPI- 8 should tend to be $100 \%$.

Calculation. The percentage should be calculated by indicating in the numerator the number of patients undergoing intervention for CRC who initiate adjuvant therapy (chemotherapy or radiotherapy) within 56 days of the index surgery (7). The denominator is the number of patients undergoing intervention for $\mathrm{CRC}$ during the reference period who initiate adjuvant therapy within 76 days (indication of the multidisciplinary panel) following the index surgical procedure.

\section{Discussion}

The continuous goal of any healthcare service is to achieve a balance between demand and supply. In recent years, it has been increasingly difficult to attain this aim; for example, in the oncology field, ageing of the population and increased research and development costs of drugs have placed considerable strain on the sustainability of healthcare. Considering this scenario, which Italy has not avoided, the methodology presented herein has the main objective of defining performance indicators that can be used to monitor critical factors in the integrated care pathway of patients with CRC, with the aim of improving the overall efficiency of care (5).

Since the public payer (e.g. healthcare service) does not coincide with the prescriber (e.g. the clinician), the process of allocating healthcare resources is often a complex exercise. The possibility of integrating tools for monitoring of the integrated care pathway constitutes an attempt to fill the information gap between prescribers and payors in the decision-making process. In this perspective, the methodology developed by the E.Pic.A. multidisciplinary group can help to better cope with increasing healthcare needs, identify inappropriate (or potentially inappropriate) activities within an integrated care pathway and thus permit reduction of waste of resources and reallocation of new resources for the access to care. The application of this methodology should increase the value of healthcare services: the better the allocation of available resources, the better the performance of structures and healthcare professionals.

Specifically, the present methodology can help to identify low-value areas of utilisation of healthcare resources and reduce the associated costs. In fact, the eight KPIs defined by the multidisciplinary E.Pic.A. panel mainly aim to identify, in the integrated care pathway for CRC, potentially inappropriate referrals for unnecessary services or anomalies in relation to the timeliness in which services are provided. In another therapeutic area (monitoring of diagnosis and treatment of patients with breast cancer), a recent experience conducted by the E.Pic.A. at the Istituto Scientifico Romagnolo per lo Studio e la Cura dei Tumori IRST of Meldola (Northern Italy) estimated that, between January 2010 and June 2016, the hospital delivered 2,516 inappropriate services for a total cost of approximately $€ 580,000$ (9). 


\section{Limitations}

The methodology used by E.Pic.A. contains some limitations that must be considered. First, analysis of the available administrative flow data may not always be suitable to correctly assess the clinical dimensions investigated by the KPIs. In fact, some targets chosen herein for the eight KPIs may not be suitable for a specific context and will therefore require a different evaluation. Second, without prejudice to the value of administrative data (real-world data) as a tool to fill the information gap of regional healthcare systems, the heterogeneity between different regions or between structures in the same region could be a further limitation of transferability of the results.

\section{Conclusion}

The adoption of E.Pic.A. methodology may help in providing a detailed picture, which is not available at present, of inappropriate and wasteful use of healthcare resources, allowing their reallocation for interventions with higher value for patients with CRC and other pathologies.

\section{Disclosures}

Financial support: This study was made possible by an educational grant from Roche S.p.A.

Conflict of interest: The authors did not receive financial support for the study. AP is a full-time employee of Roche Diagnostics S.p.A.

\section{References}

1. Spandonaro F, d'Angela D, Giordani C, Polistena B. $13^{\circ}$ Rapporto Sanità - II cambiamento della Sanità in Italia fra Transizione e Deriva del sistema. http://www.creasanita.it/13volume_dwn/ dwn_flild/Rapporto_Sanita_2017.pdf. Accessed April 16, 2019. [Article in Italian].

2. Bloomberg. Where do you get the most for your health care dollar? https://www.bloomberg.com/graphics/infographics/ most-efficient-health-care-around-the-world.html. Accessed April 16, 2019.

3. GIMBE. $2^{\circ}$ Rapporto GIMBE sulla sostenibilità del Servizio Sanitario Nazionale. June 2017. Available at: http://www. rapportogimbe.it/2_Rapporto_GIMBE_Sostenibilita_SSN.pdf. Accessed April 16, 2019. [Article in Italian].

4. Fabris $\mathrm{F}$, Bocchi $\mathrm{M}$, Mecchi $\mathrm{V}$, et al. Indicatori di esito tra gli obiettivi di budget in un'Azienda Ospedaliera Universitaria: le conoscenze dei professionisti sanitari. Evidence. 2014; 6(2):e1000069. [Article in Italian].

5. Parmenter, D. Key performance indicators: developing, implementing, and using winning KPIs. Hoboken, NJ: Wiley 2015. ProQuest Ebook Central. https://0-ebookcentral-proquest-com. opac.unicatt.it/lib/unicatt-ebooks/detail.action?doclD=1895928. Accessed April 16, 2019.

6. Associazione Italiana di Oncologia Medica (AIOM). I numeri del cancro in Italia nel 2017. http://www.registri-tumori.it/PDF/ AIOM2017/2017_numeri_del_cancro.pdf. Accessed April 16, 2019. [Article in Italian].

7. Associazione Italiana di Oncologia Medica (AIOM). Linee guida: neoplasie del retto e ano. Edizione 2018. https://www.aiom.it/ wp-content/uploads/2018/11/2018_LG_AIOM_RettoAno.pdf. Accessed April 16, 2019. [Article in Italian].
8. Programma Nazionale Esiti (PNE). https://pne.agenas.it/PNEed 16/index.php. Accessed April 16, 2019. [Article in Italian].

9. Massa I, Balzi W, Burattini C, et al. The challenge of sustainability in healthcare systems: frequency and cost of inappropriate patterns of breast cancer care (the E.Pic.A study). Breast. 2017;34:103-107. 